\title{
Low-threshold heterogeneously integrated InP/SOI laser with a double adiabatic taper coupler
}

\author{
M. Lamponi ${ }^{\mathrm{a}}$ S. Keyvaninia ${ }^{\mathrm{b}}$, C. Jany ${ }^{\mathrm{a}}$, F. Poingt ${ }^{\mathrm{a}}$, F. Lelarge ${ }^{\mathrm{a}}$, G. de Valicourt ${ }^{\mathrm{a}}$, G. Roelkens ${ }^{\mathrm{b}}$, D. Van \\ Thourhout $^{\mathrm{b}}$, S. Messaoudene ${ }^{\mathrm{c}}$, J.-M. Fedeli ${ }^{\mathrm{c}}$, G.H. Duan ${ }^{\mathrm{a}}$ Senior Member, IEEE
}

\begin{abstract}
We report on a heterogeneously integrated InP/SOI laser source realized through DVS-BCB wafer bonding. The hybrid lasers present several new features. The III-V laser is only $1.7 \mu \mathrm{m}$ wide, reducing the power consumption of the device. The silicon waveguide thickness is $400 \mathrm{~nm}$, compatible with highperformance modulator designs and allowing efficient coupling to a standard $220 \mathrm{~nm}$ high index contrast silicon waveguide layer. In order to make the mode coupling efficient, both the III-V waveguide and silicon waveguide are tapered, with a tip width for the III-V waveguide of around $800 \mathrm{~nm}$. These new features lead to good laser performance: a lasing threshold as low as $30 \mathrm{~mA}$ and an output power of more than $4 \mathrm{~mW}$ at room temperature in continous wave operation regime. Continuous wave lasing up to $70^{\circ} \mathrm{C}$ is obtained.
\end{abstract}

Index Terms - Hybrid integrated circuits, silicon laser, silicon-on-insulator (SOI) technology, adiabatic taper.

\section{INTRODUCTION}

$\mathrm{S}$ ilicon photonics is drawing increasing attention due to the promise of fabricating low-cost, compact circuits that integrate photonic and microelectronic elements [1]. Today, practical Si-based light sources are still missing, despite the recent demonstration of an optically pumped germanium laser [2]. This situation has driven research to the heterogeneous integration of III-V semiconductors on silicon. In order to densely integrate the III-V semiconductors with the silicon waveguide circuits, mainly DVS-BCB adhesive wafer bonding and molecular bonding techniques are used and are actively reported in state-of-the-art hybrid lasers [3-6]. In these approaches, unstructured InP dies are bonded, epitaxial layers down, on a SOI waveguide circuit wafer, after which the InP growth substrate is removed and the III-V epitaxial film is processed. The design space for hybrid InP/SOI lasers is large

Manuscript received July, 2011. This work was supported in part by EU Helios project.

${ }^{\mathrm{a}}$ M. Lamponi, C. Jany, F. Poingt, F. Lelarge, G. de Valicourt and G.H. Duan are with III-V Lab, a joint lab of 'Alcatel-Lucent Bell Labs France', 'Thales Research and Technology' and 'CEA Leti', Campus Polytechnique, 1, Avenue A. Fresnel, 91767 Palaiseau cedex, France (Email : marco.lamponi@35lab.fr).

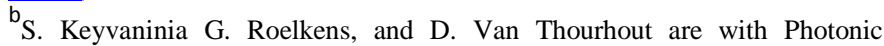
Research Group, INTEC, Ghent University-IMEC, Sint-Pietersnieuwstraat 41, B-9000 Ghent

${ }^{\text {C }}$ S. Messaoudene and J.-M. Fedeli are with III-V Lab, a joint lab of 'AlcatelLucent Bell Labs France', 'Thales Research and Technology' and 'CEA Leti', LETI, Minatec, 17 rue des Martyrs, F-38054 GRENOBLE cedex 9, France and in particular the coupling between the optical mode in the top active III-V waveguide and that in the bottom passive SOI waveguide plays an important role. In literature, two main solutions are reported. In a first solution, the silicon waveguide layer is very close to the III-V waveguide layers. In this case, the active waveguide is indeed a hybrid waveguide, with a large III-V mesa on top of the silicon waveguide. The optical mode is strongly confined in the silicon waveguide and the evanescent tail of the optical mode overlaps with the multi quantum well (MQW) layer stack [3]. This solution was originally developed for molecular bonding of III-V dies on SOI [3,4], but recently also an adhesive bonding version of these hybrid III-V/silicon lasers was presented [5]. The second solution has been specifically developed for the bonding of III-V dies on SOI using an intermediate low index bonding layer [7]. In this case, coupling between the III-V and silicon waveguide is realised by adiabatically tapering the silicon waveguide. It is to be noted that in both solutions, the silicon waveguide thickness is always larger than $500 \mathrm{~nm}$ and the III$\mathrm{V}$ effective waveguide width is quite large $(>4 \mu \mathrm{m})$. Such thick silicon waveguides are not compatible with most highperformance modulator designs, which have usually a thickness of less than $400 \mathrm{~nm}$. Moreover, the large III-V waveguide width leads to a threshold current larger than 50 $\mathrm{mA}$ in most published results for Fabry-Perot and DBR lasers. This paper reports on a hybrid laser using DVS-BCB bonding presenting several new features: narrow III-V waveguides in the range from 1.5 to $2 \mu \mathrm{m}$, thin silicon waveguide thickness $(400 \mathrm{~nm})$ and an adiabatic taper in both the III-V and silicon waveguide. These new features lead to a lasing threshold as low as $30 \mathrm{~mA}$ and an output power of more than $4 \mathrm{~mW}$ at room temperature in continous wave operation regime.

\section{Device Structure and Fabrication}

Fig. 1(a) shows the adiabatic taper coupler of the laser structure. The structure can be divided into three parts. In the center of the device the optical mode is confined to the III-V waveguide, which provides the gain. At both sides of this section there is a coupling region that couples light from the III-V waveguide to the underlying silicon waveguide. After the coupling region the light is guided by a silicon waveguide without III-V on top. 


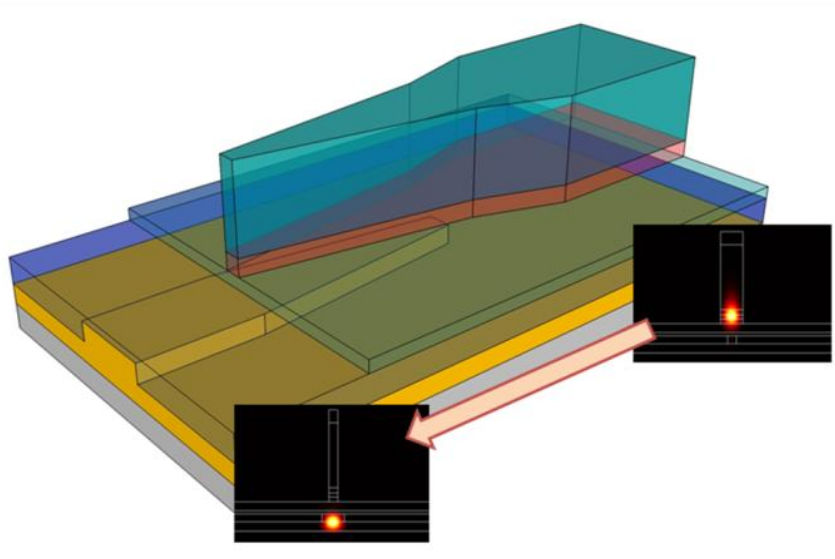

(a)

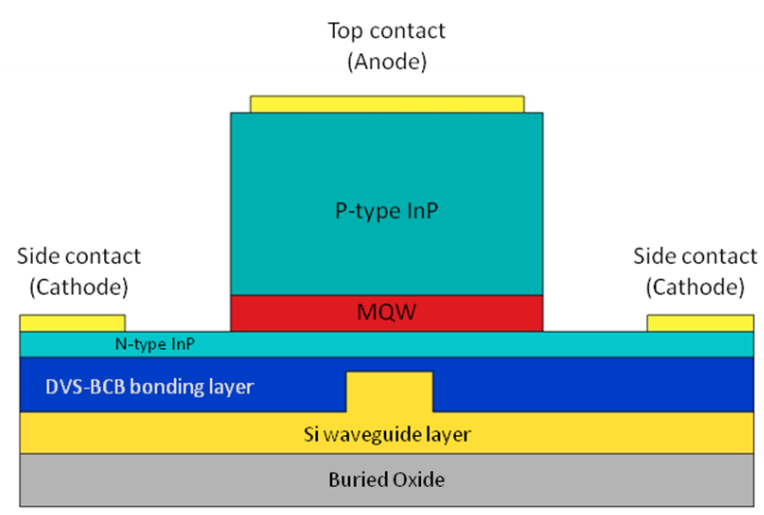

(b)

Figure 1 (a) 3D view of the coupling structure of the hybrid laser with representative mode profiles in two cross-sections (b) the detailed cross-sectional view of the center of the hybrid laser

The III-V region consists of a p-InGaAs contact layer, a p-InP cladding layer, 6 InGaAsP quantum wells surrounded by two InGaAsP separate confinement heterostructure $(\mathrm{SCH})$ layers, and an n-InP layer. The quantum wells, $8 \mathrm{~nm}$ thick, are separated by $10 \mathrm{~nm}$ thick $1.17 \mathrm{Q}-\mathrm{InGaAsP}$ barrier layers. The thickness of both $\mathrm{SCH}$ layers is $100 \mathrm{~nm}$ and the thickness of the $n$-InP layer is $200 \mathrm{~nm}$. The SOI substrate $(200 \mathrm{~mm}$ wafer manufactured by SOITEC) is composed of a 400nm monocrystalline silicon layer on top of a $2 \mu \mathrm{m}$ thick buried oxide layer on a silicon substrate. The silicon rib waveguides have a height of $400 \mathrm{~nm}$, an etch depth of $180 \mathrm{~nm}$ and a width of $1 \mu \mathrm{m}$. The III-V epitaxial layers are transferred to the patterned SOI wafer through DVS-BCB adhesive bonding. The bonding layer thickness is around $80 \mathrm{~nm}$.

\section{A. Active Waveguide}

The active waveguide structure and the guided mode profile are illustrated in Fig. 1. The active waveguide is a $1.7 \mu \mathrm{m}$ wide waveguide. The mode profiles are calculated using the mode matching method [8]. The calculated MQW optical confinement is $11.3 \%$.

\section{B. Coupling structure}

As shown in Fig. 1(a), a double taper structure allows the efficient coupling of light from the III-V waveguide to the silicon waveguide [9-10]. To achieve index matching between the two waveguides a deep ridge III-V waveguide is used in the double taper region. Figure 2 shows the coupling efficiency of the double adiabatic taper (silicon waveguide tapers from $350 \mathrm{~nm}$ to $1 \mu \mathrm{m}$, while the III-V waveguide tapers taper starts from $1 \mu \mathrm{m}$, while the DVS-BCB bonding layer thickness is $80 \mathrm{~nm}$ ) for three values of the III-V tip width: 0.4 , 0.8 and $1 \mu \mathrm{m}$. Efficient coupling can only be achieved using a tip width of $0.4 \mu \mathrm{m}$ or less. Such a requirement on the tip width is due to $400 \mathrm{~nm}$ silicon waveguide thickness. For thicker silicon waveguides a larger tip width can be tolerated.

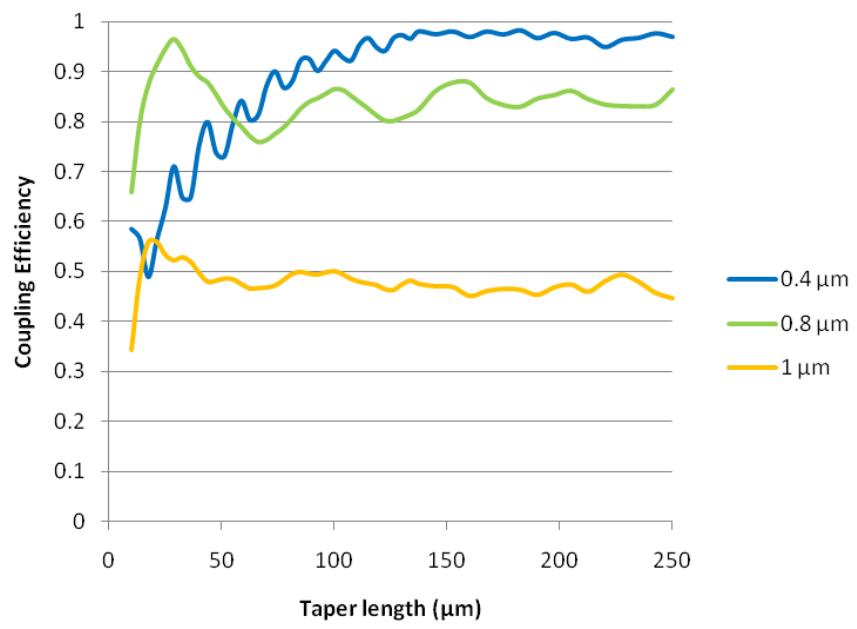

Figure 2: Coupling efficiency for different III-V taper tip widths as a function of the double taper length

\section{Fabrication}

After DVS-BCB adhesive bonding and InP substrate removal, an $\mathrm{SiO}_{2}$ hard mask was defined using 248nm UV lithography. ICP etching was used to etch through the InGaAs layer and inside the InP p-doped layer. The InP p-doped layer etching was completed by chemical selective etching. The MQW layer was etched by $\mathrm{CH}_{4}: \mathrm{H}_{2}$ RIE. Figure 3 show a scanning electron microscope picture of the III-V taper. We can see that the tip width is around $0.8 \mu \mathrm{m}$. This is due to the peculiar topology of the hybrid sample, which makes it impossible to achieve the designed dimension of the taper tips by contact lithography.

The active waveguide is encapsulated with DVS-BCB. A $\mathrm{Ti} / \mathrm{Pt} / \mathrm{Au}$ alloy was used for metallisation on both $\mathrm{p}$ and $\mathrm{n}$ sides. Finally, the III-V/silicon wafer is cleaved, to form a Fabry-Perot laser cavity. 


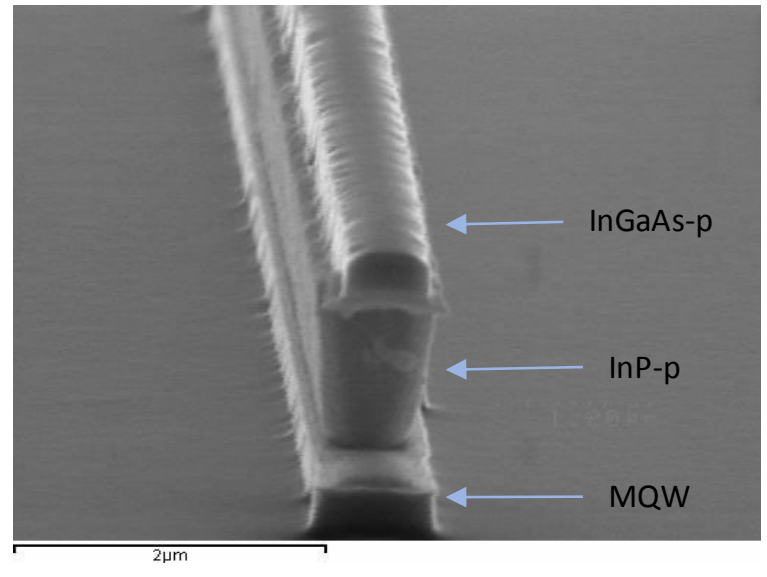

Figure 3: SEM picture of a III-V waveguide taper tip

\section{EXPERIMENTS AND RESULTS}

The device is mounted on a temperature controller set to different temperatures. The laser output is collected by a photodiode located in front of the cleaved facet. The L-I and V-I curves are shown in figure 4 for a hybrid laser with a III-V waveguide width of $1.7 \mu \mathrm{m}$. The length of the straight III-V waveguide section is $490 \mu \mathrm{m}$. The coupling section length is $230 \mu \mathrm{m}$. The overall cavity length is $1700 \mu \mathrm{m}$, including the passive silicon waveguide. The device has a threshold current of $30 \mathrm{~mA}$ and a maximum single facet continuous wave output power of $4.5 \mathrm{~mW}$ at $20^{\circ} \mathrm{C}$. At $60^{\circ} \mathrm{C}$ the laser still exhibits an output power of more than $1 \mathrm{~mW}$. The series resistance is 5 Ohms, while the slope efficiency is $0.043 \mathrm{~W} / \mathrm{A}$. Based on these results a $\mathrm{T}_{0}$ of $69 \mathrm{~K}$ was extracted and a $\mathrm{T}_{1}$ of $94 \mathrm{~K}$. The kinks in the L-I characteristics are due to the jumps of the dominant lasing mode. Figure 5 shows the optical spectrum for an injection current of $100 \mathrm{~mA}$. One can see that there are perturbations on the Fabry-Perot modes, due to parasitic reflections created by the taper tip. Considering the III-V tip width, we estimate that the coupling efficiency is around $80 \%$ and the power reflection coefficient due to the taper is $3 \%$.

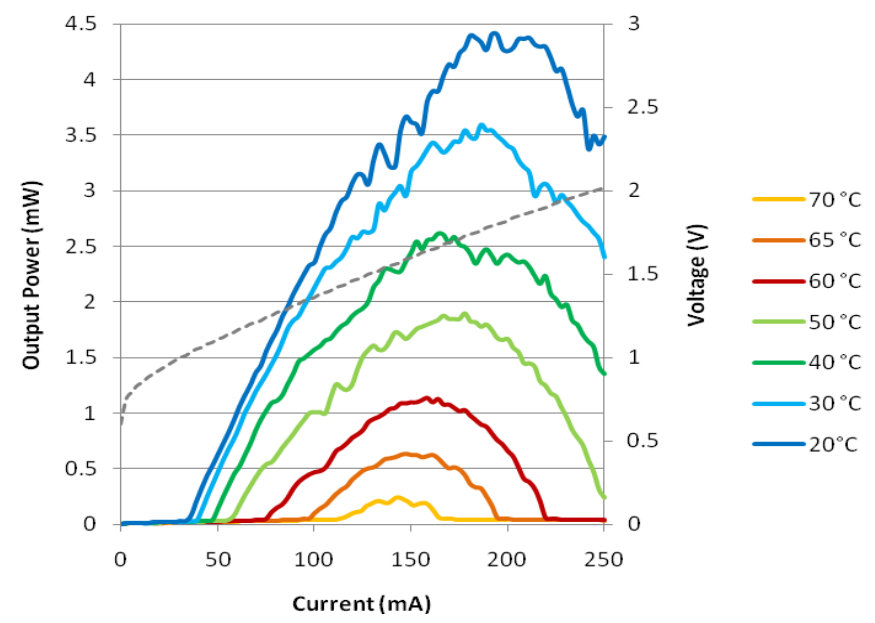

Figure 4: Continuous wave single facet laser power as a function of drive current at different stage temperatures and the (dotted) I-V curve of the laser.

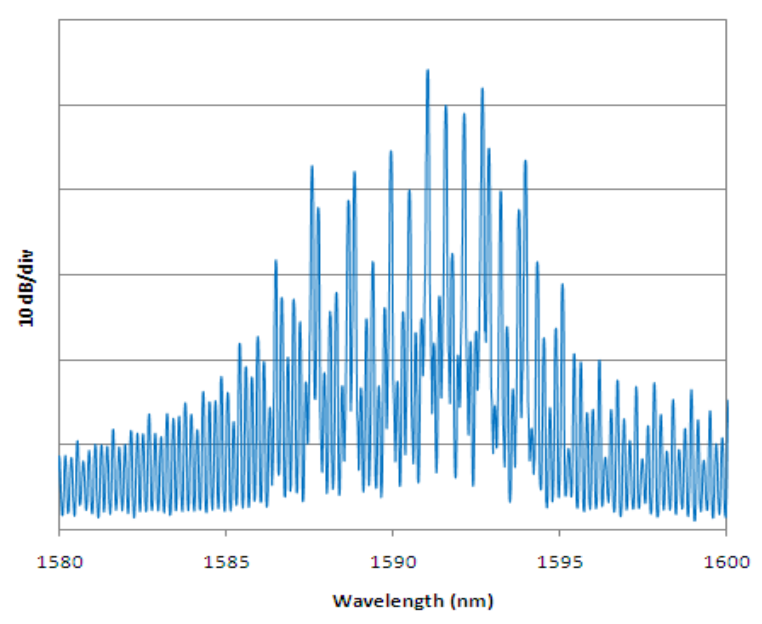

Figure 5: Lasing spectrum of the hybrid laser

\section{CONCLUSIONS}

We demonstrate a low-threshold (30mA) DVS-BCB bonded III-V on silicon laser based on the adiabatic transfer of the optical mode between the III-V waveguide layer and silicon waveguide layer. To the best of our knowledge this is the lowest threshold current value ever reported in literature for a heterogeneous III-V/silicon Fabry-Perot or DBR hybrid ridge laser. The low power consumption of this kind of device makes it suitable for access network application. Following this proof-of-principle demonstration more complex laser configuration such as DFB and DBR lasers based on the same coupling principle can be envisioned.

\section{REFERENCES}

[1] C. Gunn, "CMOS photonics for high-speed interconnects", IEEE Proceedings of Computer Science, 2006.

[2] J. Liu, et al. , "Ge-on-Si laser operating at room temperature", Opt. Lett. 35, 679-681, 2010

[3] A.W. Fang, et al. , "Hybrid silicon evanescent devices", Materials Today, Volume 10, Issues 7-8, p. 28-35, 2007.

[4] H. Park, M. N. Sysak, H.-W. Chen, A. W. Fang, D. Liang, L. Liao, B. R. Koch, J. Bovington, Y. Tang, K. Wong, M. Jacob-Mitos, R. Jones, and J. E. Bowers, "Device and Integration Technology forSilicon Photonic Transm", IEEE JOURNAL OF SELECTED TOPICS IN QUANTUM ELECTRONICS, VOL. 17, NO. 3, pp. 671-688, 2011.

[5] S. Stankovic, G. Roelkens, D. Van Thourhout, R. Jones, M. Sysak, J. Heck, "1310nm evanescent hybrid III-V/Si laser based on DVS-BCB bonding" Integrated Photonics Research, Silicon and Nano-Photonics (IPR), Canada, p.IWC3, 2011

[6] G. Roelkens, et al. , "III-V/Si photonics by die-to-wafer bonding", Materials Today, Volume 10, Issues 7-8, p.36-43, 2007.

[7] B. Ben Bakir, et al. , "Electrically driven hybrid Si/III-V lasers based on adiabatic mode transformers", Proceedings of the SPIE Conference Photonics Europe, Bruxelles, 2010.

[8] Fimmwave, Photon Design, http://www.photond.com

[9] S. Keyvaninia, et al. , "Engineering the heterogeneously integrated IIIV/SOI tunable laser", 14th Annual Symposium of the IEEE Photonics Benelux Chapter, Belgium, p.141-144, 2009.

[10] M. Lamponi, et al. ,"Heterogeneously integrated InP/SOI laser using double tapered single-mode waveguides through InP die to SOI wafer bonding", Group Four Photonics Topical Meeting, Beijing, China Sept. 2010 